Correction

\title{
Correction: Ramos-Pérez, I. et al. Calibration of Correlation Radiometers Using Pseudo-Random Noise Signals. Sensors 2009, 9, 6131-6149
}

\author{
Isaac Ramos-Pérez ${ }^{1}{ }^{1}$, Xavi Bosch-Lluis ${ }^{1}$, Adriano Camps ${ }^{1}$, Nereida Rodriguez-Alvarez ${ }^{1}$, \\ Juan Fernando Marchán-Hernandez ${ }^{1}$, Enric Valencia-Domènech ${ }^{1}$, Carlos Vernich ${ }^{2}$, \\ Sonia de la Rosa ${ }^{2}$ and Sebastián Pantoja ${ }^{2}$
}

1 Remote Sensing Lab., Dept. Signal Theory and Communications, Universitat Politècnica de Catalunya (UPC) Campus Nord, Bldg D3, Tel.: +34-934-017-362, E-08034 Barcelona, Spain; E-Mails: xavier.bosch@tsc.upc.edu (X.B.-L.); camps@tsc.upc.edu (A.C.); nereida@tsc.upc.edu (N.R.A.); jfmarchan@tsc.upc.edu (J.F.M.H.); valenzia@tsc.upc.edu (E.V.D.)

2 DAS Photonics, Ciudad Politécnica de la Innovación, Camino de Vera s/n, Edificio 8F, E-46022 Valencia, Spain; E-Mails: sonia.delarosa@dasphotonics.com (S.de la R.); cvernich@dasphotonics.com (C.V.); spantoja@dasphotonics.com (S.P.)

* Author to whom correspondence should be addressed; E-Mail: isaacramos@tsc.upc.edu (I.R.P.);

Tel.: +34-934-017-361; Fax: +34-934-017-232.

Received: 15 September 2009 / Published: 15 September 2009

It has come to our attention that in the original version of a paper published in Sensors recently [1], the names of four co-authors were misspelled with several hyphens missing. The names should be as indicated below:

Isaac Ramos-Pérez ${ }^{1}{ }^{1}$, Xavi Bosch-Lluis ${ }^{1}$, Adriano Camps ${ }^{1}$, Nereida Rodriguez-Alvarez ${ }^{1}$, Juan Fernando Marchán-Hernandez ${ }^{1}$, Enric Valencia-Domènech ${ }^{1}$, Carlos Vernich ${ }^{2}$, Sonia de la Rosa ${ }^{2}$ and Sebastián Pantoja ${ }^{2}$

\section{Reference}

1. Ramos-Pérez, I.; Bosch-Lluis, X.; Camps, A.; Rodriguez-Alvarez, N.; Marchan-Hernandez, J.F.; Valencia, E.; Vernich, C.; de la Rosa, S.; Pantoja, S. Calibration of Correlation Radiometers Using Pseudo-Random Noise Signals. Sensors 2009, 9, 6131-6149.

(C) 2009 by the authors; licensee Molecular Diversity Preservation International, Basel, Switzerland. This article is an open-access article distributed under the terms and conditions of the Creative Commons Attribution license (http://creativecommons.org/licenses/by/3.0/). 